Luciano Guimarães Artico'

JoSÉ MAURO MADI

Alessandra EIfler GuerRa Godor ${ }^{3}$

Celso Piccoli Coelho ${ }^{4}$

Renato Lú́s RomBaLDI ${ }^{5}$

Graziela Rech Artico ${ }^{6}$

Artigo original

Palavras-chav
cardiovasculares

na gravidez

Gravidez

Hipertensão/patologia

Placenta/patologia

Placenta/irrigação sanguínea

Placenta/citologia

Keywords

Pregnancy complications, cardiovascular/pathology

Pregnancy

Hypertension/pathology

Placenta/blood supply

Placenta/cytology

Placenta/pathology

\section{Alterações histopatológicas em placentas humanas relacionadas às síndromes hipertensivas}

\author{
Histopathological changes in buman placentas related \\ to bypertensive disorders
}

\section{Resumo}

OBJETIVO: determinar a prevalência de alterações histopatológicas em placentas humanas, relacionadas às síndromes hipertensivas. MÉTODOS: estudo de corte transversal, que comparou as alterações histopatológicas identificadas em 43 placentas oriundas de gestantes do grupo de hipertensas (GrHip) com as de 33 placentas de gestantes do grupo de normotensas (GrNor). Foram analisados o peso, volume e ocorrência macro e microscópica de infartos, coágulos, hematomas, aterose (obliteração parcial, espessamento de camadas e presença de vasos hialinizados) e alterações de Tenney-Parker (ausentes, discretas e proeminentes), bem como a localização de infartos e coágulos (central, periférico ou associação de ambos). Para a análise estatística foram usados os testes do $\chi^{2}$ e $t$ de Student, bem como médias, desvios padrões e percentuais. Considerou-se como significante um $\mathrm{p}<0,05$. RESULTADOS: o estudo macroscópico revelou que as placentas do GrHip, se comparadas às do GrNor, apresentaram menor peso $(461,1$ versus $572,1 \mathrm{~g})$ e volume $\left(437,4\right.$ versus $\left.542,0 \mathrm{~cm}^{3}\right)$ e percentuais aumentados de infartos $(51,2$ versus $45,5 \% ; p<0,05 ; O R=1,15)$ e de coágulos 151,2 versus 15,1\%; p<0,05; OR=5,4). Nos GrHip e GrNor, os infartos microscópicos ocorreram em 83,7 e 45,5\%; $p<0,05 ; O R=4,3$, respectivamente. A aterose e as alterações de Tenney-Parker associaram-se estatisticamente às síndromes hipertensivas $(p<0,05)$. CONCLUSÕES: os dados obtidos permitem associar menor peso e volume placentário, maior percentual de infartos macro e microscópicos, coágulos, aterose e alterações de Tenney-Parker às placentas relacionadas com gestações que cursaram com síndromes hipertensivas.

Abstract

PURPOSE: to determine the prevalence of histopathological changes, in human placentas, related to hypertensive syndromes. METHODS: a transversal study that compares histopathological changes identified in 43 placentae from hypertensive pregnant women $(\mathrm{HypPr})$, with the ones from 33 placentae from normotensive pregnant women (NorPr). The weight, volume and macroscopic and microscopic occurrence of infarctions, clots, hematomas, atherosis (partial obliteration, thickness of layers and presence of blood vessels hyalinization) and Tenney-Parker changes (absent, discreet and prominent), as well as the locating of infarctions and clots (central, peripheral or the association of both) have been analyzed. The $\chi^{2}$ and t Student tests have been used for the statistical analysis, as well as medians, standard deviations and ratios. It has been considered as significant, $\mathrm{p}<0.05$. RESULTS: the macroscopic study of HypPr placentae have presented lower weight $(461.1$ versus $572.1 \mathrm{~g})$ and volume $\left(437.4\right.$ versus $\left.542.0 \mathrm{~cm}^{3}\right)$, higher infarction (51.2 versus 45.5\%; $p<0.05$ : $O R=1.15$ ) and clots (51.2 versus 15.1\%; $p<0.05 ; O R=5.4$ ) ratios, as compared to the NorPr's. In the HypPr and NorPr, microscopic clots have occurred in 83.7 versus $45.5 \%$ l $<<0.05$; $\mathrm{OR}=4.31$, respectively. Atherosis and Tenney-Parker changes have been statistically associated to the hypertensive syndromes (p<0.05). CONCLUSIONS: the obtained data allow us to associate lower placentary weight and volume, higher ratio of macro and microscopic infarction, clots, atherosis and Tenney-Parker changes to placentae of gestations occurring with hypertensive syndromes.
Correspondência:

Luciano Guimarães Ártico Avenida Júlio de Castilhos, 1.095/602 - Centro CEP 95010-003 - Caxias do Sul (RS), Brasil E-mail: casalartico@gmail.com

Recebido

25/11/08

Aceito com modificacões

20/1/09
Serviço de Obstetrícia e Ginecologia do Hospital Geral da Universidade de Caxias do Sul - UCS - Caxias do Sul (RS), Brasil.

Médico do Ambulatório Central da Universidade de Caxias do Sul - UCS - Caxias do Sul (RS), Brasil.

2 Professor Titular da Unidade de Ensino Médico de Tocoginecologia do Centro de Ciências da Saúde da Universidade de Caxias do Sul - UCS - Caxias do Sul (RS), Brasil.

3 Professora de Anatomia Patológica do Centro de Ciências da Saúde da Universidade de Caxias do Sul - UCS - Caxias do Sul (RS), Brasil.

4 Professor Titular da Unidade de Ensino Médico de Anatomia Patológica do Centro de Ciências da Saúde da Universidade de Caxias do Sul - UCS - Caxias do Sul (RS), Brasil.

5 Preceptor da Unidade de Ensino Médico de Tocoginecologia do Centro de Ciências da Saúde de Universidade de Caxias do Sul

- UCS - Caxias do Sul (RS), Brasil.

${ }^{6}$ Acadêmica do Curso de Medicina da Universidade de Caxias do Sul - UCS - Caxias do Sul (RS), Brasil. 


\section{Introdução}

$\mathrm{Na}$ atualidade, a resposta inflamatória materna, no momento da placentação, pode ser de fundamental importância para o entendimento da etiopatogenia das síndromes hipertensivas na gestação. A compreensão desse processo também inclui o fato de que a função ótima da placenta pode ser prejudicada por inúmeros mecanismos, incluindo a hipertensão, estresse oxidativo e a ocorrência de infartos.

Devido à baixa perfusão uteroplacentária, quadros hipertensivos leves a moderados no terceiro trimestre da gravidez podem ser uma resposta adaptativa da mãe para que seja mantido fluxo sanguíneo adequado no compartimento fetal. Nessas circunstâncias, os segmentos miometriais da circulação uteroplacentária sofrem grave hiperplasia e arteriosclerose, em oposição à alta resistência e à alta pressão periférica. Com a evolução da pré-eclampsia, a aterose aguda se superpõe às artérias hiperplasiadas, ocasionando lesões vasculares graves.

No compartimento materno, as síndromes hipertensivas são caracterizadas por uma condição de hipercoagulabilidade, de vasoespasmo, coagulação intravenosa e microtrombose em diferentes órgãos ${ }^{1,2-4}$. Avaliações macroscópicas e microscópicas da placenta realizadas imediatamente após o parto permitem a identificação dos aspectos citados 5 .

Benirschke e Kaufmann ${ }^{2}$ observaram que exame macroscópico e microscópico da placenta após o secundamento proporciona ao profissional assistente razoável compreensão da vida intrauterina, principalmente se esses achados são complementados por estudo histopatológico. Os resultados podem tornar-se úteis no cuidado do recémnascido, já que permitem uma série de ilações perinatais. Além disso, podem proporcionar registros importantes a serem usados por neonatologistas e obstetras em futura assistência pré-natal ${ }^{1,2}$.

Assim, a meta deste estudo foi analisar a prevalência das alterações histopatológicas em placentas correlacionadas a gestações complicadas por síndromes hipertensivas.

\section{Métodos}

Com o objetivo de determinar a prevalência das alterações histopatológicas em placentas humanas relacionadas às síndromes hipertensivas, realizou-se estudo de corte transversal, no período de janeiro de 2003 a dezembro de 2004, no Serviço de Ginecologia e Obstetrícia do Hospital Geral da Universidade de Caxias do Sul.

Para tanto, foi realizada análise macro e microscópica de 43 placentas oriundas de gestantes hipertensas e que foram denominadas de grupo hipertenso (GrHip).
Compuseram esse grupo cinco placentas relacionadas à hipertensão prévia, treze placentas relacionadas à préeclampsia severa, dez placentas relacionadas à pré-eclampsia leve, dez placentas relacionadas à hipertensão prévia com pré-eclampsia superajuntada e cinco placentas relacionadas a casos de hipertensão gestacional. As gestantes incluídas no GrHip foram classificadas segundo o American College of Obstetrics and Gynecology ${ }^{6}$.

Para fins comparativos, foram selecionadas no mesmo período do estudo 33 placentas de gestantes normotensas (grupo normotenso - GrNor), que não apresentaram qualquer tipo de intercorrência clínica durante a gestação. Com vista à eliminação de possível viés, considerou-se o tabagismo como critério de exclusão para ambos os grupos.

Todas as gestantes foram orientadas e informadas quanto ao objetivo do estudo, requisitando-se, por ocasião do seu recrutamento, a assinatura do termo de consentimento livre e esclarecido. O projeto foi aprovado pela Comissão de Ética em Pesquisa da Universidade de Caxias do Sul.

Após o secundamento, as 76 placentas foram encaminhadas ao Serviço de Anatomia Patológica para avaliação macroscópica (peso, volume, identificação do percentual das áreas de infartos e coágulos) e microscópica (percentual de infartos e hematomas, aterose e alteração de Tenney-Parker, bem como suas localizações), segundo rotina e questionário específicos (Figuras 1 a 8). As alterações de Tenney-Parker são representativas de hipertrofia do sinciciotrofoblasto e são consideradas lesões típicas da pré-eclampsia. Caracterizam-se pela formação de proeminentes nós sinciciais e numerosos aglomerados de núcleos nos vilos, que sinalizam o envelhecimento precoce da placenta (Figuras 6 a 8).

Previamente à aferição do peso e do volume placentário, o cordão umbilical foi seccionado junto à sua base, na face fetal da placenta, e as membranas removidas de todo o disco. O peso foi verificado sempre com a mesma balança digital marca Toledo, São Paulo. Para se analisar o volume, as placentas foram imersas em um copo tipo Becker com 3,4 litros de água e a quantidade extravasada era avaliada num tubo volumétrico.

As placentas foram imersas em formol a $10 \%$ por um período médio de 24 a 48 horas para fixação. Após esse tempo, eram selecionados os cortes para o processamento histotécnico. Os cortes utilizados na avaliação microscópica foram randomizados, extraindose porções dos discos placentários dos terços periférico, intermediário e central (dois cortes de cada área), além de um corte do cordão e um da membrana. Cada membrana placentária foi enrolada e fixada dessa forma para ser cortada em seguida. Quando se observavam lesões macroscópicas, eram realizados mais dois cortes 


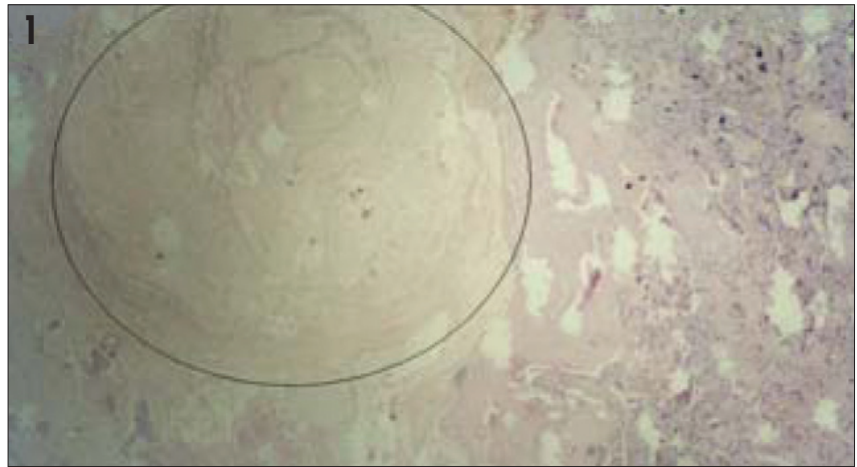

Figura 1 - Fotomicrografia de área de infarto vilositário (circunscrito). Tecido adjacente normal (aumento de 100X HE).

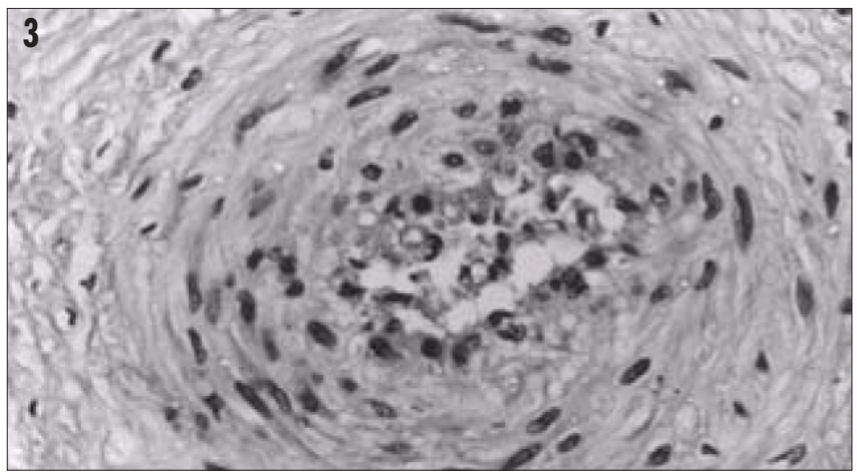

Figura 3 - Fotomicrografia demonstrando vaso viloso com infiltração de leucócitos e espessamento da parede do vaso (aumento de 400X HE).

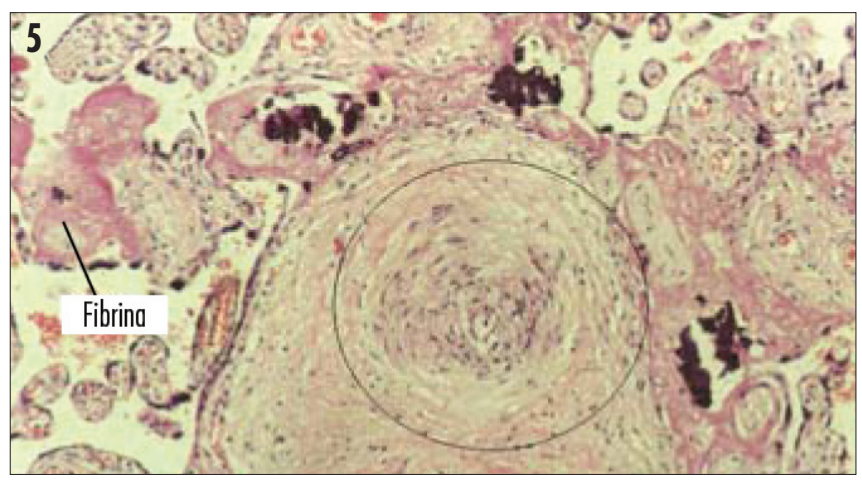

Figura 5 - Fotomicrografia demonstrando vilo com aterose e obliteração parcial da luz do vaso (circunscrito maior), vaso viloso sem obstrução (circunscrito menor) e fibrina intervilositária (aumento de 100X HE).

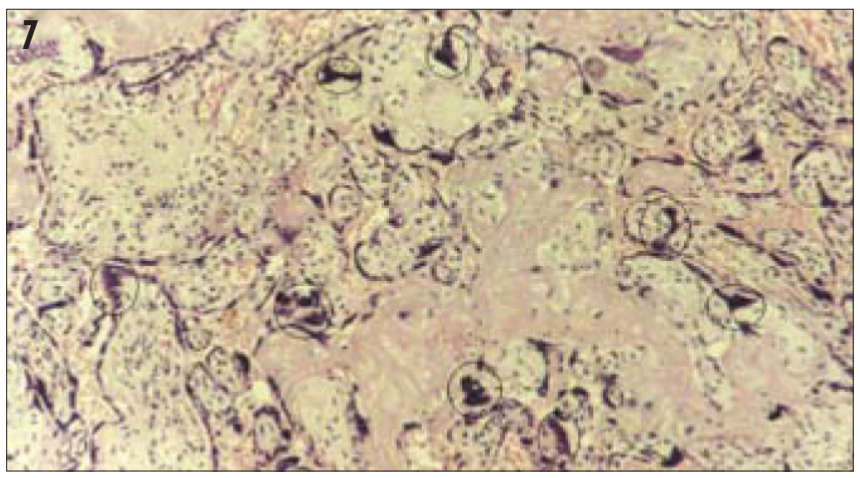

Figura 7 - Fotomicrografia demonstrando calcificações e alterações de Tenney-Parker (aumento de 100X HE).

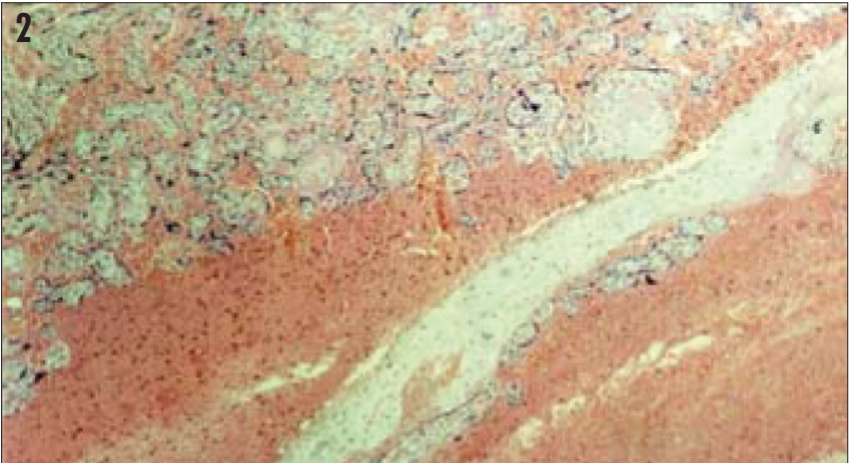

Figura 2 - Fotomicrografia demonstrando hematoma no disco placentário adjacente ao tecido normal. Membrana amniótica entre áreas de hematoma (aumento de 100X HE).

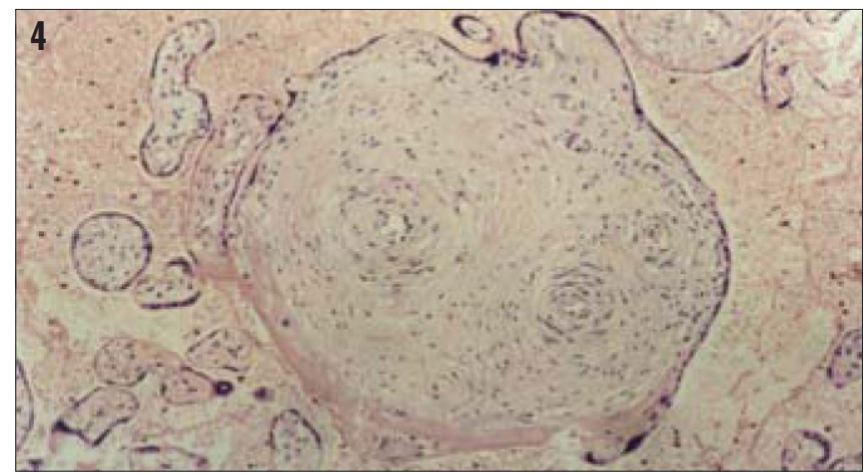

Figura 4 - Fotomicrografia demonstrando aterose e depósito de fibrina, com obliteração parcial (aumento de 100X HE).

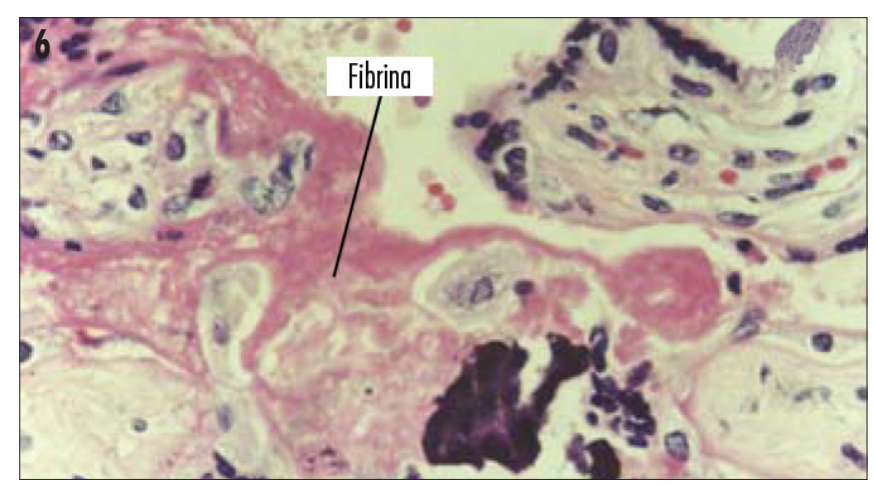

Figura 6 - Fotomicrografia demonstrando calcificação e fibrina intervilositária (aumento de 400X HE).

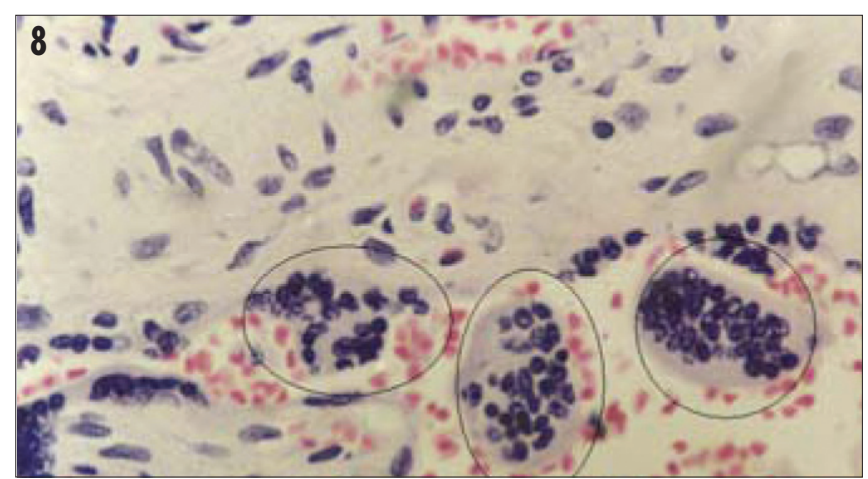

Figura 8 - Fotomicrografia demonstrando alterações de Tenney-Parker (circunscritas) (aumento de 400X HE). 
em cada área afetada. Utilizou-se método padrão de embebição em parafina, com coloração pela técnica da hematoxilina-eosina ${ }^{7}$. Em seguida, os cortes histológicos foram examinados ao microscópio óptico, sempre pelo mesmo observador. Nos casos de identificação de intercorrências graves em ambos os grupos durante a gestação, excluíam-se as pacientes do estudo a fim de se evitarem vieses de aferição.

As informações obstétricas e perinatais foram inseridas no Statistical Package for the Social Sciences for Personal Computer 12.0 (SPSS-PC) e as avaliações estatísticas foram realizadas mediante a aplicação do teste não-paramétrico do $\chi^{2}$ com correção de Yates (ocorrência de aterose, infartos, coágulos, hematomas e alterações de Tenney-Parker) e do teste $t$ de Student (peso, volume, percentuais de infartos e coágulos). Adotou-se o nível de significância de $5 \%$.

\section{Resultados}

Ao exame macroscópico, as placentas das pacientes incluídas nos GrHip e GrNor apresentaram peso médio de 461,1 versus $572,1 \mathrm{~g}(\mathrm{p}<0,05)$ e volume placentário médio de 437,4 e $542 \mathrm{~mL}(\mathrm{p}<0,05)$, respectivamente. Pode-se identificar o menor peso e volume nas placentas oriundas do GrHip, característica de gestações submetidas à vasoconstricção promovida pela hipertensão.

$\mathrm{Na}$ face placentária materna, nos grupos em estudo, a média de coágulos identificados foi de 5,5 e 1,7\% $(\mathrm{p}<0,05)$, enquanto que o percentual médio de infartos foi de 9,8 versus $3,9 \%(\mathrm{p}<0,05)$, respectivamente (Tabela 1 e Figura 1).

À microscopia, as placentas dos grupos hipertenso e normotenso apresentaram percentual de infartos (Figura 1) de 9,6 versus $3,2 \%(\mathrm{p}<0,05)$ e percentual de hematomas (Figura 2$)$ de 5,3 versus $5 \%(\mathrm{p}<0,05)$, respectivamente (Tabela 1 e Figura 1).

Ao se compararem os GrHip e GrNor, a microscopia pôde constatar a ocorrência de infartos placentários em
$83,7 \%(\mathrm{n}=36)$ versus $45,5 \%(\mathrm{n}=15) \mathrm{p}<0,005$; IC95\% $=1,3-$ 14,3; OR=4,29, respectivamente (Tabela 2).

Os diferentes aspectos histológicos da aterose (Figura 3), como obliteração parcial (Figuras 4 e 5), espessamento das camadas e presença de vasos hialinizados (Figura 6), puderam ser observados em 44,2\% $(\mathrm{n}=19)$ versus $3,1 \%(\mathrm{n}=1), 44,2 \%(\mathrm{n}=19)$ versus $3,1 \%$ $(\mathrm{n}=1)$ e $27,9 \%(\mathrm{n}=12)$ versus $3,1 \%(\mathrm{n}=1)$, respectivamente $(\mathrm{p}<0,000001)$ nos grupos GrHip e GrNor (Tabela 2).

A avaliação macroscópica dos infartos, segundo as suas localizações no disco placentário, constatou que a localização periférica da lesão foi a mais incidente no GrHip $(25,6 \% ; n=11)$ e no GrNor $(33,3 \% ; n=11)$, respectivamente $\mathrm{p}<0,0008$; IC95\% $=0,4-32 ; \mathrm{OR}=1,15)$. Quanto à localização dos coágulos no disco placentário, observou-se distribuição semelhante, ou seja, a lesão periférica também foi mais incidente no GrHip $(25,6 \% ; \mathrm{n}=11)$ e no GrNor $(9,1 \% ; \mathrm{n}=3), \mathrm{p}<0,000001$; IC95\% $=1,6-19,4 ;$ OR $=5,4(1,6-19,4)$, conforme apresentado na Tabela 3 .

Nas placentas dos GrHip e GrNor, as alterações de Tenney-Parker (Figuras 7 e 8 ) discretas e proeminentes foram observadas à microscopia em $69,8 \%(\mathrm{n}=30)$ versus $6,1 \%(\mathrm{n}=2)$, respectivamente $(\mathrm{p}<0,00001)$, conforme a Tabela 3 .

Tabela 1 - Distribuição das médias das variáveis macroscópicas e microscópicas placentárias

\begin{tabular}{lrrr}
\hline Variáveis & GrHip & GrNor & Valor de p \\
\hline Macroscópicas & & & \\
Peso (g) & 461,1 & 572,1 & $<0,05$ \\
Volume (mL) & 437,4 & 542,0 & $<0,05$ \\
Infartos (\%) & 9,8 & 3,9 & $<0,05$ \\
Coćgulos (\%) & 5,5 & 1,7 & $<0,05$ \\
Microscópicas & & & \\
Infartos (\%) & 9,6 & 3,2 & $<0,05$ \\
Hematomas (\%) & 5,3 & 0,5 & $<0,05$ \\
\hline
\end{tabular}

GrHip=gestantes do grupo de hipertensas; GrNor=gestantes do grupo de normotensas.

Tabela 2 - Distribuição da ocorrência microscópica de infartos placentários e dos aspectos histológicos da aterose

\begin{tabular}{|c|c|c|c|c|c|c|}
\hline \multirow{2}{*}{ Variáveis } & \multicolumn{2}{|c|}{$\begin{array}{l}\text { GrHip } \\
\mathrm{n}=43\end{array}$} & \multicolumn{2}{|c|}{$\begin{array}{l}\text { GrNor } \\
\mathrm{n}=33\end{array}$} & \multirow{2}{*}{ Valor de $\mathrm{p}^{*}$} & \multirow{2}{*}{ OR (IC95\%) } \\
\hline & $\begin{array}{c}\text { Sim } \\
\text { n (\%) }\end{array}$ & $\begin{array}{c}\text { Não } \\
\text { n (\%) }\end{array}$ & $\begin{array}{c}\text { Sim } \\
\text { n (\%) }\end{array}$ & $\begin{array}{c}\text { Não } \\
\text { n (\%) }\end{array}$ & & \\
\hline Infartos placentários & $36(83,7)$ & $7(6,3)$ & $15(45,5)$ & $18(54,5)$ & $<0,005$ & 4,29 (1,3-14,3) \\
\hline \multicolumn{7}{|l|}{ Aterose } \\
\hline Obliteração parcial & $19(44,2)$ & $24(55,8)$ & $1(3,1)$ & $32(96,9)$ & $<0,000001$ & \\
\hline Vasos hialinizados & $12(27,9)$ & $31(72,1)$ & $1(3,1)$ & $32(96,9)$ & $<0,000001$ & \\
\hline
\end{tabular}

*Teste do $\chi^{2}$ com correção de Yates; GrHip=gestantes do grupo de hipertensas; GrNor=gestantes do grupo de normotensas. 
Tabela 3 - Distribuição dos infartos e coágulos segundo suas localizações (estudo macroscópico) e alterações de Tenney-Parker, segundo a intensidade

\begin{tabular}{|c|c|c|c|c|c|c|}
\hline Variáveis & \multicolumn{2}{|c|}{$\begin{array}{l}\text { GrHlip } \\
n=43 \\
n(\%)\end{array}$} & \multicolumn{2}{|c|}{$\begin{array}{c}\text { GrNor } \\
n=33 \\
n(\%)\end{array}$} & \multirow[t]{2}{*}{ Valor de $\mathrm{p}^{*}$} & OR (IC95\%) \\
\hline Infartos & & & & & & $1,15 \quad(0,4-3,2)$ \\
\hline Ausentes & 21 & $(48,8)$ & 18 & $(54,5)$ & $<0,001$ & \\
\hline Centrais & 4 & $(9,3)$ & 2 & $(6,1)$ & $<0,000001$ & \\
\hline Periféricos & 11 & $(25,6)$ & 11 & $(33,3)$ & $<0,0008$ & \\
\hline Ambos & 7 & $(16,3)$ & 2 & $(6,1)$ & $<0,000001$ & \\
\hline Coágulos & & & & & & $5,4 \quad(1,6-19,4)$ \\
\hline Ausentes & 21 & $(48,8)$ & 28 & $(84,8)$ & $<0,004$ & \\
\hline Centrais & 4 & $(9,3)$ & 1 & $(3,0)$ & $<0,000001$ & \\
\hline Periféricos & 11 & $(25,6)$ & 3 & $(9,1)$ & $<0,000001$ & \\
\hline Ambos & 7 & $(16,3)$ & 1 & $(3,0)$ & $<0,000001$ & \\
\hline $\begin{array}{l}\text { Alterações de } \\
\text { Tenney-Parker }\end{array}$ & & & & & $<0,00001$ & \\
\hline Ausentes & 13 & $(30,2)$ & 31 & $(93,9)$ & & \\
\hline Discretas & 19 & $(44,2)$ & 2 & $(6,1)$ & & \\
\hline Proeminentes & 11 & $(25,6)$ & & - & & \\
\hline
\end{tabular}

*Teste do $\chi^{2}$ com correção de Yates; ambos=central+periférico; GrHip=gestantes do grupo de hipertensas; GrNor=gestantes do grupo de normotensas.

\section{Discussão}

Estudos de placentas correlacionadas às gestantes préeclâmpticas e hipertensas prévias demonstram volumes e pesos reduzidos, bem como aumento do percentual de áreas de infarto e hematomas ${ }^{5,8}$. Neste estudo, são constatadas essas alterações. À microscopia, esses infartos apresentamse como tecido vilositário necrótico; do ponto de vista macroscópico, correspondem a áreas grosseiramente triangulares, de dimensões variáveis, relacionadas à placa basal e de localização, sobretudo, periférica no disco placentário ${ }^{2}$. No presente estudo, quando da análise macroscópica, os infartos periféricos foram os predominantes, tanto no GrHip (25,6\%) quanto no GrNor $(33,3 \%)$.

O infarto, embora não seja lesão específica da préeclampsia, já que também é encontrado em gestações de termo e sem complicações, aparece com mais frequência e em maior extensão na região periférica. Alguns autores consideraram como potencialmente patológicos os infartos centrais e paracentrais, os infartos marginais em número superior a três, ou cujo diâmetro ultrapasse $4 \mathrm{~cm}^{2,3,5}$.

Em placentas obtidas de gestantes portadoras de diferentes tipos de hipertensão, foram identificados infartos macroscópicos em $61 \%$ dos casos, acometendo uma área superior a aproximadamente $5 \%$ de tecido placentário em $16 \%$ delas $^{8}$. No presente estudo, observou-se a lesão em 51,2\% dos casos, sendo 9,3\% (n=4) central, 25,6\% $(\mathrm{n}=11)$ periférico e $16,3 \%$, quando da associação central e periférico $(n=7)$, como se observa na Tabela 3 .

Alguns autores têm citado aumento significante de infartos placentários em síndromes hipertensivas, enfatizando ser comum a sua localização no centro do disco placentário. Observaram, também, significante correlação com baixos índices de Apgar e baixo peso no nascimento ${ }^{1,9}$. Do ponto de vista macroscópico, observou-se no presente estudo 51,2\% ( $\mathrm{n}=22)$ de infartos no GrHip versus $45,5 \%$ no GrNor.

Em um estudo observou-se que a lesão esteve presente em $33 \%$ das mulheres com forma leve da doença e em $60 \%$ das formas graves, sendo que nos casos graves a área infartada tendeu a ser maior, envolvendo 5 a $10 \%$ do parênquima placentário ${ }^{2}$. Entre os casos do presente trabalho, à macroscopia, os percentuais de lesões provocadas por infartos nas placentas dos grupos hipertenso e normal foram de 9,8 versus $3,9 \%$, respectivamente, enquanto à microscopia, foram observadas $83,7 \%$ de placentas do GrHip com essa lesão específica. No GrNor, a lesão foi observada em $45,5 \%$ dos casos.

O descolamento prematuro da placenta costuma ocorrer em uma área de infarto, podendo caracterizar-se desde muito pequenos, detectados somente por ultrassonografia, até muito grandes, causando dor e interrupção da gestação 5 . O tecido vilositário pode ser comprimido pelos hematomas, gerando infartos e hipoxia. Quando a síndrome hipertensiva é a causa do descolamento, ocorre hemorragia, iniciada nas lesões vasculares deciduais. A trombose das arteríolas deciduais pode levar à necrose decidual e, consequentemente, à hemorragia venosa ${ }^{2}$. É citado que a ruptura das artérias espiraladas comprometidas pela trombose provocam o descolamento 9 . O hematoma retroplacentário ocorre em aproximadamente $45 \%$ das placentas, sendo três vezes mais frequentes em mulheres portadoras de pré-eclampsia ${ }^{2}$. A etiopatogenia dessa lesão é bastante discutível, estando, nos casos de hipertensão, provavelmente relacionada à ruptura de vasos espiralados maternos, previamente lesados ${ }^{2}$. São observados coágulos em 51,2 e 15,1\% nas placentas dos GrHip e GrNor, respectivamente.

Entre os relacionados às lesões obstrutivas das artérias deciduais, que ocorrem em pacientes hipertensas, são separados os casos de grávidas com pré-eclampsia das portadoras de hipertensão prévia ${ }^{2}$. Tais lesões, independentemente do tipo de hipertensão, estariam associadas ao menor fluxo sanguíneo dependente do espasmo arteriolar generalizado, decorrendo daí o estado hipoxêmico. É sugerido que o déficit de oxigênio demonstra que a circulação placentária da paciente hipertensa está diminuída em um terço e confirma que a saturação de oxigênio nas artérias e veias umbilicais do feto de grávidas com pré-eclampsia está diminuída ${ }^{2}$. Indubitavelmente, a oclusão parcial ou total dos vasos placentários cumpre papel importante na gênese do comprometimento conceptual ${ }^{2}$.

Embora a hipertensão arterial essencial ocasione lesões morfológicas sistêmicas tardiamente, também induz à 
proliferação do tecido conectivo nas paredes das artérias basais, além de determinar proliferação excessiva do tecido fibroso e muscular liso tanto na íntima como na média, promovendo estenose ou até oclusão das mesmas. Em pacientes com pré-eclampsia e eclâmpsia foram observadas necrose fibrinoide aguda e aterose aguda ${ }^{2,10}$. A aterose engloba várias alterações microscópicas que podem estar associadas ou não: obliteração parcial vascular, espessamento da parede vascular, infiltração leucocitária na parede vascular, reação granulomatosa, presença de hialinização vascular, substituição da íntima por macrófagos e diminuição da luz dos vasos ${ }^{2,8}$. No estudo em questão, quando do aparecimento de aterose, a obliteração parcial da luz dos vasos foi observada em 44,2 versus $3,1 \%$, o espessamento das camadas em 44,2 versus $3,1 \%$ e a presença de vasos hialinizados em 27,9 versus 3,1\%, nos GrHip e GrNor, respectivamente. Esses achados mostraram-se similares aos de outra pesquisa, que observou o espessamento das camadas em $41,7 \%$ das placentas analisadas ${ }^{2}$.

É relatado acúmulo de fibrina na íntima dos vasos, levando-os à oclusão. É citado que também a hialinização e a necrose da parede vascular acoplada ao depósito de macrófagos estão presentes em duas condições maternas: pressão sanguínea elevada e vasculite ou infecção bacteriana decidual ${ }^{2,8,10}$. Nenhuma das gestantes incluídas nos grupos estudados foi acometida por quaisquer tipos de infecções, e por isso se aceita que as alterações observadas tenham sido exclusivamente provocadas pelas síndromes hipertensivas. Em uma série de casos, a aterose esteve relacionada às formas graves de hipertensão, verificandose o comprometimento vascular em todos os níveis de gravidade das patologias hipertensivas ${ }^{4,5}$.

Foram observadas em placentas de gestantes hipertensas lesões características nas arteríolas dos troncos vilosos, representadas por hiperplasia da camada muscular e forte espessamento do tecido conjuntivo subendotelial, com proliferação fibrocitária e invasão leucocitária, que originou progressiva obstrução da luz dos vasos ${ }^{2}$. As lesões se distinguiam das diagnosticadas em placentas de pacientes normais, ao termo da gestação. Aqui, o fato dominante foi a esclerose que atingiu as faces maternas e fetais, os troncos vilosos e as vilosidades. Dessa forma, a aterose tornou-se a marca da doença hipertensiva na gestação, fazendo crer que as principais alterações vistas na placenta são resultado das modificações nas artérias espiraladas. A Tabela 2 enfatiza a ocorrência da aterose, em todos os seus níveis, nas placentas do grupo hipertenso.

Em 1940, Tenney e Parker ${ }^{11}$ publicaram um estudo em que a hipertrofia do sinciciotrofoblasto é uma lesão típica da pré-eclampsia. A lesão era caracterizada pela formação de proeminentes nós sinciciais e numerosos aglomerados de núcleos nos vilos, que sinalizavam o envelhecimento precoce da placenta. Embora alguns desses nós pudessem ser identificados em placentas de termo, originadas de gestações normais, apresentavam-se muito mais proeminentes em placentas relacionadas à doença hipertensiva, mesmo em estágios precoces da hipertensão. As alterações de Tenney-Parker podem ser vistas em número significativo após a $32^{\mathrm{a}}$ semana de gestação, estando presentes, no termo, em aproximadamente um terço das vilosidades ${ }^{11}$. Embora haja polêmica em torno do seu significado, estudos de microscopia eletrônica têm demonstrado se tratar de células envelhecidas, agrupadas sob a forma de nós, enquanto as células mais novas, oriundas do citotrofoblasto, permaneceriam dispersas na superfície do vilo (Figura 4). O aumento do número de nós sinciciais, associado à necrose dos vilos, depósito de fibrina e focos de calcificação, fariam parte do quadro de envelhecimento fisiológico da placenta, incrementado por ocasião de uma síndrome hipertensiva ${ }^{1,4,9}$. Assim, o fenômeno caracterizado pelo aumento das alterações de Tenney-Parker é tido hoje como reflexo de um regime de hipoxia, seja ele uteroplacentário, como nos casos de eclâmpsia, ou feto-placentário, como nos casos de tromboses de artérias fetais. Foi dividida a ocorrência das alterações de Tenney-Parker em discretas e proeminentes. No grupo hipertenso, as discretas foram observadas em 19 casos $(44,2 \%)$, e no normotenso, em dois casos $(6,1 \%)$. As alterações proeminentes foram observadas em 11 casos $(25,6 \%)$ no grupo hipertenso.

Assim, os dados obtidos permitem associar a presença de maior percentual de infartos, coágulos, hematomas, aterose e alterações de Tenney-Parker, bem como menor peso e volume placentários, com as síndromes hipertensivas. A inclusão do estudo rotineiro macro e microscópico das placentas deve ser incentivado para que se consigam maiores esclarecimentos sobre o funcionamento do leito placentário durante o desenvolvimento fetal, em gestações patológicas e normais. Destino desconhecido ou incineração do material placentário deve ser evitado, de forma que seja possível assegurar os dados necessários a futuras correlações neonatais. Observa-se que as alterações macro e microscópicas caracterizadas nas placentas que cursaram com diferentes estágios das gestações hipertensas não são apanágios desse ou daquele tipo de hipertensão arterial, podendo essas alterações ser identificadas em todos os quadros hipertensivos.

\section{Agradecimento}

Agradecemos ao professor Petrônio Fagundes de Oliveira Filho pela revisão estatística. 


\section{Referências}

1. Corrêa RRM, Salge AKM, Ribeiro GA, Ferraz MLF, Reis MA, Castro ECC, et al. Alterações anatomopatológicas da placenta e variações do índice de Apgar. Rev Bras Saúde Matern Infant. 2006;6(2):239-43.

2. Benirschke K, Kaufmann P. Pathology of the human placenta. $4 a$ ed. New York: Springer; 2000.

3. Corrêa RR, Gilio DB, Cavellani CL, Paschoini MC, Oliveira FA, Peres LC, et al. Placental morphometrical and histopathology changes in the different clinical presentations of hypertensive syndromes in pregnancy. Arch Gynecol Obstet. 2008;277(3):201-6.

4. Granger JP, Alexander BT, Llinas MT, Bennett WA, Khalil RA. Pathophysiology of hypertension during preeclampsia linking placental ischemia with endothelial dysfunction. Hypertension. $2001 ; 38(3$ Pt 2):718-22.

5. Majumdar S, Dasgupta $H$, Bhattacharya K, Bhattacharya A. A study of placenta in normal and hypertensive pregnancies. J Anat Soc India. 2005;54(2) 1-9.
6. ACOG Committee on Practice Bulletins - Obstetrics. ACOG practice bulletin. Diagnosis and management of preeclampsia and eclampsia. Number 33, January 2002. Obstet Gynecol. 2002;99(1):159-67

7. Michalany J. Métodos selecionados de colorações e de impregnações argênticas. In: Michalany J, editor. Técnica histológica em anatomia patológica: com instrução para cirurgião, enfermeira e citotécnica. São Paulo: Michalany; 1998. p. 125-81.

8. Janthanaphan M, Kor-Anantakul O, Geater A. Placental weight and its ratio to birth weight in normal pregnancy at Songkhlanagarind Hospital. J Med Assoc Thai. 2006;89(2): 130-7.

9. Mesquita MRS, Sass N, Stavalle JN, Camano L. O leito placentário no descolamento prematuro da placenta. Rev Bras Ginecol Obstet. 2003;25(8):585-91.

10. Huppertz B. Placental origins of preeclampsia: challenging the current hypothesis. Hypertension. 2008;51 (4):970-5.

11. Tenney B, Parker F. The placenta in toxemia of pregnancy. Am J Obstet Gynecol. 1940;39:1000-5. 